\title{
Development of a Strengths Measurement Scale for the lifestyle transformation process
}

\author{
Reiko Okahisa", and Toshiko Tada ${ }^{2}$ \\ ${ }^{1}$ Major in Health Sciences, Graduate School of Health Sciences, the University of Tokushima, \\ Tokushima, Japan, ${ }^{2}$ Department of Community Nursing, Major in Nursing, Institute of Health Bio- \\ sciences, the University of Tokushima Graduate School, Tokushima, Japan
}

\begin{abstract}
This study aimed to develop a Strengths Measurement Scale (SMS) for assessment of people in the process of transforming their own lifestyle, and to examine the reliability and validity of the SMS. This is to establish a foothold for introducing the Strengths Model into health guidance for the prevention of lifestyle-related diseases. We conducted an anonymous, voluntary questionnaire survey with our SMS comprising 38 items proposed in a preliminary investigation. In total, 1,339 Japanese adults participated, and responses from 1,160 participants (effective response rate of $86.6 \%$ ) were analyzed. We calculated Cronbach's alpha coefficient for testing the reliability. To test the validity, we examined the construct validity through exploratory factor analyses. Based on the results of the factor analysis performed on the 38 question items of the SMS, we adopted four factors and 36 question items. Cronbach's alpha coefficients of all the factors were 0.80 or above. There were significant, positive correlations between the SMS and the Modified Perceived Health Competence Scale (PHCS), and between the SMS and the Adolescent Resilience Scale (ARS). The reliability and validity of the SMS for the lifestyle transformation process were confirmed, suggesting that the scale is applicable to health guidance for preventing lifestyle-related diseases. J. Med. Invest. 61 : 84-93, February, 2014
\end{abstract}

Keywords : lifestyle transformation process, Strengths Measurement Scale (SMS), reliability, validity, health guidance

\section{INTRODUCTION}

Lifestyle-related diseases are an important issue that requires evidence-based health guidance focusing on primary prevention. This type of health guidance emphasizes outcome evaluation and aims to change a person's lifestyle (1), which eventually leads to prevention of lifestyle-related diseases, the extension of healthy life expectancy and promotion

Received for publication December 2, 2013 ; accepted December 16, 2013.

Address correspondence and reprint requests to Reiko Okahisa, Department of Community Nursing, Major in Nursing, Institute of Health Biosciences, the University of Tokushima Graduate School, 3-18-15 Kuramoto-cho, Tokushima 770-8509, Japan and Fax : +81-88-633-9977. E-mail : reiko.okahisa@tokushima-u. ac.jp of a person's wellbeing.

Unfortunately, changing a person's deep-rooted lifestyle is never easy. In health guidance, it is important not only to aim for outcomes focusing on numerical targets, but also to holistically grasp individuals as citizens in society, maintain a perspective that utilizes the autonomy and individuality of people, and provide support for these processes $(2,3)$.

In the Specific Health Guidance (SHG) prepared by Ministry of Health, Labour and Welfare, its Standard Program for Health Check and Guidance (the finalized version) states (1), "Health guidance to be implemented should emphasize individuality with attention to recipients' vision and behavioral transformation stage (the preparation state)." However, 
there is not yet an indicator to comprehensively grasp and objectively evaluate the total personality, autonomy and individuality of health guidance recipients. Consequently, health guidance tends to depend on an individual health trainer's subjective judgment and experiences.

The "Strengths Model" is a concept to represent an individual's holistic views, autonomy and individuality (4).

The Model is a social constructivist way of understanding human powers and abilities (hereinafter "Strengths"), focusing on subjective experiences. This concept originated from the change from the pathological model to the life model (5), and has been developed in the field of social welfare mainly for people with disabilities or sickness and for senior citizens (5-9). However, none of the studies on primary prevention, which considers approaches for disease prevention and promotion of health, focus on Strengths. Consequently, there is no indicator to objectively evaluate an individual's Strengths.

According to a previous study, Strengths include multiple interacting factors that contribute to the quality of people's lives and their accomplishments and satisfaction ; these Strengths serve as a source of empowerment (4). Health guidance for preventing lifestyle-related diseases is considered to have the same aim as that of Strengths. Furthermore, Rapp et al. (4) stated that everyone has Strengths based on the concept of "recovery", meaning that people cure and recover themselves by defining themselves as individuals. Hazama defines Strengths as the power to create changes (10) ; that is, people reflect on themselves while going through relationships and interactions with others. Moreover, the meanings they give to events determine whether those events become their own Strengths. The consequent transformation of awareness generates further changes. Accordingly, we consider that even in the process of lifestyle transformation, Strengths act as a profound drive that generates changes.

The aim of this study is to develop a Strengths Measurement Scale (SMS) for assessment of people in the process of transforming their own lifestyle, and to examine the reliability and validity of the SMS.

\section{DEFINITION OF TERMS IN THIS STUDY}

Lifestyle transformation process : The process of people undergo to restructure their lives and to work toward wellbeing for the purpose of health promotion and preventing lifestyle-related diseases.

Strengths: The powers and abilities of people in the lifestyle transformation process.

Health guidance : Support for people in the lifestyle transformation process.

\section{MATERIALS AND METHODS}

We conducted a preliminary investigation and a main investigation to complete the development of the SMS for the lifestyle transformation process, and to test its reliability and validity.

\section{Subjects}

The participants were Japanese adults aged between 20 and 64 years old chosen through random sampling. A total of 1,339 adults participated in the main study, and we received responses from 1,229 of them (collection rate, 91.8\%).

\section{Survey method}

We conducted an anonymous questionnaire survey from August to October 2013. The questionnaire sheets were directly distributed in cooperation with the participants' offices and training institutes. The collection method was voluntary submission from the participants.

\section{Questionnaire items}

(1) Basic attributes : age, gender, employment status, marital status, the presence/absence of chronic diseases

(2) Items related to lifestyle and health (11) : breakfast, snacking, nutritional balance, salt content, drinking, smoking, exercise, sleep, weight, behavioral transformation stage $(12,13)$, the presence/ absence of regular health checkup

(3) SMS : We used the draft version of the SMS consisting of 63 question items in a preliminary investigation. We conducted the main investigation with the main version SMS comprising 38 items proposed in a preliminary study

\section{Creation of the draft version SMS}

Twenty-five Japanese adults (16 men and 9 women) were asked during interviews to narrate their experiences of changing lifestyle. From the interview data, we extracted the parts in which they spoke of their Strengths, and performed a qualitative inductive study. Six categories and 68 codes were 
extracted.

The six categories were treated as the sub-concepts of the scale's constructive concept, "Strengths in the lifestyle transformation process": [Strengths to view one's own life with a long-term perspective], [Strengths to recognize one's own existence in interactions with others], [Self-awareness that one can improve one's own lifestyle if there are opportunities], [Strengths to handle and control stress], [Strengths to analyze one's own life and tendency] and [Strengths to reconstruct oneself through trial and error] (14). We created 63 question items from codes based on the categories.

Regarding the content validity of the scale, we referred to opinions from one researcher in developing the scale and three specialists in community nursing. On the basis of those opinions, we examined the validity of the question items, the clarity of expressions used and the easiness for the participants to answer the questions.

Preliminary investigation : Creation of the main version of the SMS

A total of 272 Japanese adults aged between 20 and 64 years old (mean age $41.9 \pm$ SD 10.8) participated in the preliminary investigation, and we received responses from 210 of them (return rate of $77.2 \%)$; responses from 207 participants (139 men and 68 women ; effective response rate, $76.1 \%)$ were analyzed.

Regarding the Item-Total correlations analysis (I-T analysis), one of the items, "Sometimes, I can allow myself to do something unhealthy," showed a value of 0.124 , and thus was excluded. No item showed any ceiling or floor effect.

i. Exploratory factor analysis

Factor analyses (principal factor method, promax rotation) were performed on the 62 items after excluding one item through the item analysis. The results of a scree plot indicated the factor number of four. However, there were six categories in the sub-concept of the SMS's constructive concept, "Strengths in the life transformation process," which was obtained from the qualitative inductive study. Further, the number of question items was no less than 62 . As a result, we set the factor number as five. We deleted those items with factor loadings of 0.45 or less. Moreover, we reviewed the contents of those question items whose factor loadings were high in relation to multiple factors, deleted question items one by one to see changes in the factor loadings, and finally determined which question items were to be excluded. In relation to the question items of "I can utilize past failures for the future" and "I know what I can do and what I cannot do," although their factor loadings were 0.409 and 0.324 , respectively, we left these items at the time of the preliminary study as they were considered to be distinctive items for Strengths in the lifestyle transformation process. We repeated factor analyses and selected 38 question items consisting of five factors, for which a proper factor structure was obtained in terms of contents. The cumulative contribution ratio before rotation was $58.16 \%$, and the Spearman's correlation coefficients between the five factors were within the range of $\rho=0.337$ to 0.589 . Accordingly, there were significant, positive correlations between the five factors.

ii. Naming of subscales

Next, we interpreted the contents of the selected subscale items of the five factors. The first factor (11 items) was composed of Strengths leading to lifestyle transformation through the utilization of resources, such as "I know how to get information for maintaining my own health" and "I can consult with a specialist for my health on my initiative." We named the first factor [Utilization]. The second factor (11 items) was composed of Strengths to reconstruct one's life with a long-term perspective while coping with stress, such as "I know how to release my stress," "I can imagine my future health state" and "When I embark on things, I can start with and pile up small steps." We named the second factor [Reconstruction]. The third factor (6 items) was composed of Strengths to recognize one's own existence in interactions with others, such as "I have a person on whom I can rely," "I have a person who can support my health life" and "I know my role in my family." We named the third factor [Connectedness]. The fourth factor (5 items) was composed of Strengths to recognize one's own tendency, such as "I know my preference" and "I know my own set of values." We named the fourth factor [Self-awareness]. The fifth factor (5 items) was composed of Strengths to utilize past experiences to transform one's own lifestyle, such as "I know from experience that lifestyle improvements have an effect on my mentality" and "I can utilize my past failures for the future." We named the fifth factor [Experience].

\section{The main version of the SMS}

Based on the results in the preliminary investigation, we used the main version of the SMS containing 38 question items after reducing the 
number of question items in the draft version of the SMS from 63 to 38. Cronbach's alpha coefficient (hereinafter "alpha coefficient") of all 38 items of the main research version of SMS was 0.942 , and the alpha coefficients of the five factors were in the range of 0.832 to 0.909 .

Each question item was scored using a five-point Likert scale (Strongly agree, Agree, Neutral, Disagree and Strongly disagree). A score of five to one was allocated to the five choices, respectively. A higher score indicates higher Strengths.

(4) The scale for convergent validity

i. The modified Perceived Health Competence Scale (PHCS) (15)

Our study employed the modified Japanese version of the PHCS, which is a scale for measuring self-efficacy in the field of health management (15, 16). This scale is based on a five-point system, consisting of eight items.

\section{ii. Adolescent Resilience Scale (ARS) (17)}

The present study employed the ARS, whose reliability and validity had been tested and which has been widely utilized as a resilience scale in Japan. It is a five-point scale, consisting of 21 items.

iii. The Japanese version of the SF- $8^{\mathrm{TM}}$ Health Survey $(\mathrm{SF}-8)(18,19)$

This scale has been widely utilized as a measure of health-related quality of life and can measure eight aspects in relation to health. This is a five- or six-point scale consisting of eight items, with which physical summary scores and mental summary scores can be determined.

\section{Analytical methods}

(1) Item analysis : analysis on the ceiling and floor effects, I-T analysis

(2) Testing of reliability : calculating the alpha coefficients of each factor and the whole scale

(3) Testing of validity

i. Construct validity

We employed exploratory factor analyses (principal factor method, promax rotation). The criteria for determining the number of factors were an eigenvalue of 1.0 or more and a cumulative contribution ratio of $50 \%$ or more. Further, the criterion for determining subscale items was a factor loading of 0.45 or less. We compared the adopted factor structure with the six categories extracted at the time of developing the draft version of the SMS.

ii. Convergent validity

We calculated and examined the correlation coefficients of the SMS with the PHCS, with the ARS and with the SF-8 (by employing the Spearman rank-correlation coefficients). We set the total of the item scores in each of the SMS subscales as a subscale score, and examined correlations between the total SMS score and the total score of each of the scales above.

iii. Criterion-related validation (concurrent validity)

The relationships between the SMS and the lifestyle and the behavioral transformation stage were analyzes by using the Spearman rank-correlation coefficients.

(4) We compared the SMS scores according to the basic attributes of the participants (Mann-Whitney U test).

\section{Ethical consideration}

Approval for this study was obtained from the ethics committee of Tokushima University Hospital (No. 1316).

The following details were explained to the participants in writing: (1) the purpose and methods of the study ; (2) that their participation was voluntary and refusal of participation would attract no disadvantage ; (3) that the data would not be used for purposes other than the study ; and (4) that best efforts would be made in order to protect their identity when study outcomes were presented. We took their responses to and submission of the questionnaire sheets as their consent to participate in the study.

\section{RESULTS}

Among 1,229 questionnaire sheets collected, we excluded those sheets containing missing values in items about age, gender or lifestyle, or in the behavioral transformation stage, the main version of the SMS, the PHCS, the ARS, or the SF-8. Further, we excluded those sheets in which the responses to all the scales were "3". Accordingly, 1,160 questionnaire sheets were considered valid responses and used as analysis data (valid response rate of $86.6 \%$ ).

\section{Characteristics of the participants}

The mean age of the participants was $40.3 \pm$ SD 10.7 years old. Regarding gender, there were 631 men (54.4\%) and 529 women (45.6\%). Regarding marital status, 390 of the participants were unmarried (33.6\%), 745 were married (64.2\%) and 25 answered "Other" (divorced, etc.) (2.2\%). In relation to the presence/absence of chronic diseases, 174 of the 
participants had chronic diseases (15.0\%), while 986 $\operatorname{did}$ not (85.0\%) (Table 1).

\begin{tabular}{lcc} 
Table $1 \quad$ Characteristics of the participants & $(\mathrm{n}=1,160)$ \\
\hline Age(Mean, SD) & 40.3 & 10.7 \\
\hline \hline Basic attributes & $\mathrm{n}$ & $\%$ \\
\hline Gender & & \\
$\quad$ Men & 631 & 54.4 \\
$\quad$ Women & 529 & 45.6 \\
Employment status & & \\
Full-time & 1,014 & 87.4 \\
Part-time & 104 & 9.0 \\
$\quad$ Others & 42 & 3.6 \\
Marital status & & \\
Unmarried & 390 & 33.6 \\
Married & 745 & 64.2 \\
Others & 25 & 2.2 \\
Chronic diseases & & \\
Yes & 174 & 15.0 \\
No & 986 & 85.0 \\
\hline
\end{tabular}

\section{SMS for the lifestyle transformation process}

(1) Item analysis

In the I-T analysis, a positive correlation coefficient of 0.361 was found in relation to "I know what I can do and what I cannot do," but we left the item in the questionnaire. We did not eliminate any of the other 37 items as their correlation coefficients were between $\rho=0.425$ and 0.660 . No ceiling or floor effect was observed in all the items.

(2) Exploratory factor analysis

Table 2 presents the results of the exploratory factor analyses. We conducted factor analyses on 38 question items and determined the number of factors as four on the basis of comparisons with scree plots and factor interpretations of the five factors from the preliminary investigation. The items under the factor of [Experience] in the preliminary investigation were found under the factor of [Utilization] in the main investigation. After the second factor analysis, we excluded the subscale items of "If I want to know something, I can access necessary information on my own" (factor loading of 0.311) and "I know what I can do and what I cannot do" (factor loading of 0.281 ). After these processes, we selected four factors and 36 question items, for which a proper factor structure was obtained in terms of contents, and created the final version of the SMS. As in the Main investigation, the four factors were named [Utilization], [Reconstruction], [Connectedness] and [Self-awareness].

The cumulative contribution ratio before rotation was $56.63 \%$, and the Spearman's correlation coefficients between the four factors were within the range of $\rho=0.353$ to 0.492 , showing significant, positive correlations between the factors $(\mathrm{P}<0.01)$.

(3) Testing of reliability (Table 2 )

i. Internal consistency

The alpha coefficient of all 36 items of the final version of the SMS was 0.941, and the alpha coefficients of the four factors were in the range of 0.876 to 0.926 .

(4) Testing of validity

i. Testing of construct validity

We compared the four factors of [Utilization], [Reconstruction], [Connectedness] and [Self-awareness] adopted from the exploratory factor analyses above, with the subscale items of the five categories found in the preliminary investigation. From this, three subscale items under the factor [Experience] at the time of the preliminary investigation were included in the factor of [Utilization], and the other three factors were composed of the same items.

ii. Testing of convergent validity $(\mathrm{P}<0.01)$ (Table 3 ).

The correlation coefficient between the total SMS score and the PHCS was $\rho=0.495$. In relation to the correlations of the PHCS with the SMS subscales, the correlation with [Reconstruction] was $\rho=0.445$, that with [Utilization] was $\rho=0.434$, that with [Connectedness] was $\rho=0.294$ and that with [Self-awareness] was $\rho=0.224$.

The correlation coefficient between the total SMS score and the ARS was $\rho=0.520$. In relation to the correlations of the ARS with the SMS subscales, the correlation with [Reconstruction] was $\rho=0.637$, that with [Connectedness] was $\rho=0.353$, that with [Selfawareness] was $\rho=0.349$ and that with [Utilization] was $\rho=0.319$.

Furthermore, the correlation coefficient between the SF- 8 (mental health) and the total SMS score was $\rho=0.222$, and that between the former and the subscale [Reconstruction] was $\rho=0.325$. There was almost no correlation between the SF- 8 (physical health) and the SMS.

iii. Criterion-related validation (concurrent validity)

The correlation coefficient between the total SMS score and the lifestyle was $\rho=0.350$, and that between the former and the behavioral transformation stage was $\rho=0.366$. Almost no correlation was found between the age of the participants and the final 
Table 2 Exploratory factor analyses of Strengths Measurement Scale for the lifestyle transformation process $(n=1,160)$

\begin{tabular}{|c|c|c|c|c|}
\hline \multirow{2}{*}{ Factor/item } & \multicolumn{4}{|c|}{ Factor loading } \\
\hline & Factor1 & Factor2 & Factor3 & Factor4 \\
\hline \multicolumn{5}{|l|}{ Factor 1 : Utilization $(\alpha=0.926)$} \\
\hline 1 I have the knowledge required to maintain my health & .857 & -.093 & -.029 & .025 \\
\hline I know how to get information for maintaining my own health & .770 & -.120 & -.046 & .067 \\
\hline I can look back on my past health statuses with reference to figures relating to my health & .749 & -.069 & -.039 & .046 \\
\hline I can select information necessary for maintaining my own health & .746 & -.076 & .008 & .071 \\
\hline I put up my antenna to gather information on a routine basis & .732 & -.012 & -.037 & .031 \\
\hline I can look back on my past lifestyle with reference to figures relating to my health & .717 & -.057 & -.050 & -.045 \\
\hline I can actively create opportunities for improving my own lifestyle & .712 & .117 & .071 & -.143 \\
\hline I disseminate information regarding health to people around me & .698 & -.058 & .056 & -.054 \\
\hline I use "my ideal image of myself" as a trigger to improve my lifestyle & .683 & .039 & .044 & -.106 \\
\hline 10 I can consult with a specialist (such as a medical expert) for my health on my own initiative & .654 & -.049 & .024 & -.038 \\
\hline 11 I know from experience that lifestyle improvements have an effect on my physicality & .581 & .074 & -.031 & .158 \\
\hline 12 I think I can continue improving my lifestyle & .568 & .194 & .036 & -.083 \\
\hline 13 I know from experience that lifestyle improvements have an effect on my mentality & .542 & .138 & -.007 & .143 \\
\hline 14 I know from experience that lifestyle improvements have an effect on my sociability & .506 & .181 & -.030 & .140 \\
\hline \multicolumn{5}{|l|}{ Factor $2:$ Reconstruction $(\alpha=0.894)$} \\
\hline 15 I can cut through stress & -.132 & .850 & -.069 & .016 \\
\hline 16 I can recover my physicality and mentality after a difficult situation & -.111 & .823 & .008 & -.022 \\
\hline 17 I can choose an appropriate method for releasing stress according to situation & -.106 & .812 & -.020 & .031 \\
\hline 18 I can think of alternative plans at the time of difficulty & -.004 & .738 & .012 & -.028 \\
\hline 19 I can think out ways to effortlessly accomplish my objectives & .075 & .659 & .002 & -.060 \\
\hline 20 When taking actions, I can relate my objectives to my favorite things & .017 & .598 & .026 & .066 \\
\hline 21 I know how to release my stress & -.076 & .594 & -.035 & .158 \\
\hline 22 I can imagine my future life & .235 & .563 & .058 & -.133 \\
\hline 23 I can imagine my future health state & .287 & .528 & .037 & -.108 \\
\hline 24 When I embark on things, I can start with and pile up small steps & .052 & .467 & .039 & -.030 \\
\hline 25 I can utilize my past failures for the future & .029 & .443 & .053 & .116 \\
\hline \multicolumn{5}{|l|}{ Factor 3 : Connectedness $(\alpha=0.876)$} \\
\hline 26 I have a person on whom I can rely & -.013 & -.027 & .889 & -.108 \\
\hline 27 I have a person whom I encourage and by whom I am encouraged & -.079 & -.003 & .873 & .033 \\
\hline 28 I have a person who understands me & -.037 & .011 & .850 & .037 \\
\hline 29 I have a person who evaluates my conduct & -.078 & .047 & .723 & .150 \\
\hline 30 I have a person who can support my health life & .131 & .028 & .534 & -.088 \\
\hline 31 I know my role in my family & .176 & -.026 & .489 & .119 \\
\hline \multicolumn{5}{|l|}{ Factor 4: Self-awareness ( $\alpha=0.878)$} \\
\hline 32 I know my areas of interest & .019 & .019 & -.071 & .856 \\
\hline 33 I know my own set of values & -.008 & .026 & -.029 & .845 \\
\hline 34 I know my own personality & -.042 & -.042 & .059 & .803 \\
\hline 35 I know my preference & .075 & -.097 & .163 & .675 \\
\hline 36 I know my special skills & -.004 & .286 & -.060 & .538 \\
\hline
\end{tabular}

principal factor method, promax rotation, Cronbach's alpha coefficient $(\alpha)$ for the total score was 0.941

version of the SMS.

(5) Comparison of the SMS scores according to the basic attributes

In a gender comparison, compared with the male participants, the female participants had high total SMS scores and scored high in the subscales of
[Utilization], [Connectedness] $(\mathrm{P}<0.01)$ and [Selfawareness] $(\mathrm{P}<0.05)$, showing significant differences. No significant difference was found in relation to [Reconstruction]. Further, according to marital status, compared with the unmarried participants, the married participants had high total SMS scores 
Table 3 : Correlations among all variables

$(\mathrm{n}=1,160)$

\begin{tabular}{|c|c|c|c|c|c|c|c|c|}
\hline & Age & lifestyle & $\begin{array}{c}\text { Behavioral } \\
\text { transformation stage }\end{array}$ & SMS & PHCS & ARS & $\begin{array}{l}\text { SF-8(physical } \\
\text { summary) }\end{array}$ & $\begin{array}{l}\text { SF-8(mental } \\
\text { summary) }\end{array}$ \\
\hline Age & 1.000 & & & & & & & \\
\hline lifestyle & $.088^{\star \star}$ & 1.000 & & & & & & \\
\hline Behavioral transformation stage & $.112^{\star *}$ & $.315^{\star \star}$ & 1.000 & & & & & \\
\hline SMS & $.112^{\star *}$ & $.350^{\star *}$ & $.366^{\star *}$ & 1.000 & & & & \\
\hline PHCS & $.067^{\star}$ & $.412^{\star \star}$ & $.165^{\star \star}$ & $.495^{\star \star}$ & 1.000 & & & \\
\hline ARS & -.032 & $.134^{\star \star}$ & $.160^{\star \star}$ & $.520^{\star \star}$ & $.352^{\star *}$ & 1.000 & & \\
\hline SF-8(physical summary) & $-.115^{\star *}$ & $.098^{* *}$ & -.021 & $.123^{\star *}$ & $.202^{\star \star}$ & $.141^{\star *}$ & 1.000 & \\
\hline SF-8(mental summary) & .052 & $.095^{\star *}$ & .041 & $.222^{\star *}$ & $.220^{\star *}$ & $.318^{\star \star}$ & $-.118^{\star *}$ & 1.000 \\
\hline
\end{tabular}

Speaman's correlation coefficient : ${ }^{* \star} . \mathrm{P}<0.01,{ }^{*} . \mathrm{P}<0.05$

and scored high in the subscales of [Utilization] and [Connectedness], showing significant differences $(\mathrm{P}<0.01)$.

Furthermore, compared with those participants without chronic diseases, those with chronic diseases scored high in the subscale of [Utilization], showing significant differences $(\mathrm{P}<0.01)$.

\section{DISCUSSION}

This study aimed to develop a SMS for the assessment of people in the lifestyle transformation process, with the eventual goal of introducing the Strengths Model into health guidance for preventing lifestyle-related diseases and promoting wellbeing. The final version of the SMS comprises four subscales and 36 items.

(1) Reliability of the SMS

The alpha coefficient of all 36 items of the final version of the SMS was 0.941 , and the alpha coefficients of the four factors were in the range of 0.876 to 0.926 . This indicated that the SMS possesses a certain level of internal consistency, thereby ensuring its reliability.

(2) Validity of the SMS

To test the validity of the SMS, the construct validity and criterion-related validity were examined as follows.

i. Construct validity

The five factors adopted from the exploratory factor analyses in the preliminary investigation, [Utilization], [Reconstruction], [Connectedness], [Selfawareness] and [Experience], consisted of almost the same items as those in the six categories found in the qualitative inductive analysis at the stage of the draft SMS development. Further, in the comparison between the four factors of [Utilization],
[Reconstruction], [Connectedness] and [Self-awareness] adopted in the main study and the five factors in the preliminary investigation, [Experience] of the preliminary investigation was included in the interpretation of [Utilization] of the main study. The other three factors were composed of the same items. Accordingly, we consider that the construct validity of the SMS is ensured.

In relation to the subscale item "I can utilize my past failures for the future," although it showed a factor loading of 0.44 , which was lower than the criterion of 0.45 , it was a high value as it exceeded 0.40 and was considered to be an important item for lifestyle reconstruction. Consequently, we did not exclude this item.

ii. Convergent validity

Relationships with the PHCS, ARS and SF-8

Previous studies clarified the relationship between behavioral transformation and self-efficacy $(20,21)$. Further, in a qualitative inductive study $(3,4)$ for developing the main-study version of the SMS, one of the six categories of Strengths in the lifestyle transformation process included [Self-awareness that one can improve one's own lifestyle if there are opportunities]. From these findings, we considered the possibility of employing a Self-efficacy Scale as a scale for validating concurrent validity.

The Japanese version of the modified PHCS (15, 16) used in our main study is a self-efficacy scale in the field of health management. Shimizu (22) used the Japanese version of the modified PHCS developed by Togari et al. $(15,16)$ to examine psychological factors influencing the preventive health behavior of university students, and found the significant effectiveness from "Health Competence" to "Preventive Health Behavior". From the results of our study, the correlation coefficient between the total score of the 36 items of the final SMS and the 
PHCS was $\rho=0.495$, showing a positive correlation. Accordingly, we obtained results supporting the relationship between Strengths and self-efficacy.

In the main investigation, the correlation coefficient between the ARS and the final SMS was $\rho=$ 0.520 , and that between the ARS and the subscale [Reconstruction] was $\rho=0.637$, together showing positive correlations. Strengths are a concept similar to resilience $(23,24)$, and both originated from the change from the pathological model to the life model $(5,10)$. Furthermore, Strengths are described as a premise of resilience (23). In previous studies, Lundman $e t$ al. developed the Inner Strength Scale, tested its validity by employing a resilience scale and found a significant, positive correlation between the two scales (25). From these findings as well as the correlation between the SMS for the lifestyle transformation process and the ARS, we consider that the concurrent validity of the SMS is ensured.

Subsequently, we employed the SF- 8 as a method to test the validity and found a low correlation between the SMS and mental health. However, as there was almost no correlation between the SMS and physical health, Strengths are considered to relate mainly to mental health.

iii. Criterion-related validation (concurrent validity)

The correlation between the SMS and lifestyle was $\rho=0.350$, and that between the former and the behavioral transformation stage was $\rho=0.366$. These showed low, positive correlations between the SMS and lifestyle and behavioral transformation stage. The correlation between Strengths and lifestyle suggests the possibility that the Strengths Model can be introduced into the lifestyle transformation process. Further, the positive correlation between Strengths and the behavioral transformation stage representing the preparation state of lifestyle improvements indicates that changes in people's consciousness are associated with their Strengths in the lifestyle transformation process.

From the fact that there was almost no correlation between age and the SMS, we consider that the SMS does not relate to age.

\section{Significance of this study}

In this study, we developed the SMS for the lifestyle transformation process, examined its reliability and validity, and analyzed its structure using factor analyses. The results suggest the usability of the SMS as a Strengths Scale to be used to introduce the Strengths Model into health guidance for preventing lifestyle-related diseases and promoting wellbeing, and to comprehensively grasp people's total personality, autonomy and individuality.

In the discipline of nursing, the Inner Strength Scale (ISS) (in Sweden) and Inner Strength Questionnaire Version 4 (ISQ) (in USA) were developed in $2011(25,26)$. Although their reliability and validity have been tested, further tests are necessary in terms of their psychological features. Further, most of the previous studies on Inner Strength focus on women with chronic diseases and senior citizens $(27,28)$. Consequently, we consider that the significance of our study lies in the fact that we have developed a new scale, the SMS for the lifestyle transformation process, rather than employing the existing ISS or ISQ ; this development was based on the constructive concept that matched the purpose of the study.

In the comparison of the factor structure of SMS with ISQ and ISS in previous studies, [Connectedness] has been included in the configuration factors in all scales and is considered the essential factor of Strengths. Furthermore, SMS's [Self-awareness] was the factor with similar meaning to that of "Engagement" of ISQ and "Firmness" of ISS. However, SMS's [Utilization] and [Reconstruction] had the same naming as ISQ's "Anguish and Searching" and "Movement", as well as ISS's "Flexibility" and "Creativity", but their contents were different. In other words, it has become clear that these two factors had not only the recognition side but also the action side in long-term perspective and that they were characteristic factors in SMS.

As there was no correlation between the SMS and age, we also consider that the SMS has a high level of versatility.

The Strengths Model has been developed mainly for secondary and tertiary prevention in the field of social science. Its targets are people with disabilities or the elderly. This study has highlighted the usability of the SMS for the lifestyle transformation process, and will likely lead to the application of the Strengths Model in health guidance for preventing lifestyle-related diseases and in primary prevention for promoting wellbeing.

\section{Prospects and issues}

In this study, we developed the SMS for the lifestyle transformation process and ensured its reliability and validity. The study suggests the possibility of introducing the Strengths Model into health guidance for preventing lifestyle-related diseases. 
The following points are potential future issues.

1) With the use of the SMS developed in this study, means of support can be considered that enable health guidance recipients to reflect on and recognize their own Strengths and utilize their Strengths for lifestyle transformation.

2) By accumulating studies on Strengths according to basic attributes, the characteristics of subjects' Strengths could be clarified according to their attributes, and effective supporting methods that focus on their Strengths could be developed.

3) Ways to develop a simpler SMS or an intervention method of health guidance that utilizes the SMS could be found by further examining the relationships among the SMS and the feelings of satisfaction and empowerment.

4) Ways to utilize the SMS as an objective evaluation indicator for health trainers can be considered.

\section{CONFLICT OF INTEREST}

None of the authors have any conflicts of interest to declare.

\section{ACKNOWLEDGEMENT}

This study was supported by JSPS KAKENHI (Grant Number 24593440).

The authors would like to express our deep appreciation to those who cooperated in this study. The authors would like to extend our great thanks to Professor Hiroko Shimizu (Faculty of Medicine, Kagawa University) for useful advice. We also wish to thank Professor Tadashi Kondo (the University of Tokushima Graduate School) for helpful comments about data analysis. Lastly, we would like to thank Professor Mari Haku (the University of Tokushima Graduate School) for her consideration.

Some parts of this study were presented in the International Council of Nurses Conference in Melbourne in May 2013.

\section{REFERENCES}

1. Health, Labour and Welfare Ministry, Health Service Bureau: The standard program for health check and health guidance (the finalized version), 2007 (in Japanese)

2. Shimoda $\mathrm{T}$ : Integrated promotion project of regional healthcare 2008 population approach promotion and evaluation report. Japan Public Health Association, 2009 (in Japanese)

3. Okahisa R, Tada T, Fujii C, Matsushita Y: Health guidance method understood through role-playing after recording of daily activities among nursing university students. JNI 14 (1) : 71-77, 2011 (in Japanese)

4. Rapp CA, Goscha, RJ, Tanaka H : The strengths model, case management with people with psychiatric disabilities. Kongo-Shuppan, Tokyo, 2008 (in Japanese)

5. Kamiyama $\mathrm{H}$ : A Strengths Perspective Based on Generalist Approach to Social Work : A view of community care support. Bulletin of the Faculty of Human and Social Services, Yamanashi Prefectural University $1: 1-10,2006$ (in Japanese)

6. Yamaguchi $\mathrm{M}$ : The meaning of the study in strengths- oriented social work process. The Review of Welfare Society, 4/5 : 97-114, 2004 (in Japanese)

7. Kamiyama $\mathrm{H}$ :Applying and development strengths perspective : promotion and support of healthy life for the elderly in the community. Bulletin of Faculty of Human and Social Services, Yamanashi Prefectural University 2 : 1930, 2007 (in Japanese)

8. Okumura $\mathrm{K}$ : A study on the effectiveness of strengths based on case management : support of community living for people with slight intellectual disabilities. Japanese Journal of Social Welfare 50 (1) : 134-147, 2009 (in Japanese)

9. Shirasawa $\mathrm{M}$ : Care management based on the Strengths Model. Minerva-Shobo, Kyoto, 2009 (in Japanese)

10. Hazama K : Strengths point of view / Social constructionism / Empowerment - aid view of social welfare. Tsutsui Shobo, Tokyo, 2001 (in Japanese)

11. Morimoto $\mathrm{K}$ : The preventive medicine of the stress crisis-the viewpoint of lifestyle, NHK Books, Tokyo, 1997 (in Japanese)

12. Prochaska JO, Velicer WF : The Transtheoretical model of health behavior change. American Journal of Health Promotion 12 (1) : 38-48, 1997

13. Prochaska JO, Redding CA, Evers KE : The transtheoretical model and stages of change. In : Glanz K, Rimer BK, Viswanath K, eds. Health behavior and health education. theory, study, and practice. 4th ed. Jossey-Bass, San Francisco.CA, 2008, pp. 97-121 
14. Okahisa $\mathrm{R}$ : The strengths of the recipients of health guidance for the improvement of lifestyle habits. Major in Health Sciences, Graduate School of Health Sciences, the University of Tokushima, Tokushima, (Dissertation) 2011 (in Japanese)

15. Togari T, Ikezaki S, Yamazaki Y, Ito M, Zenko A, Taguchi R: The development of Perceived Health Competence Scale (PHCS) Japanese version. Journal of Health and Human Ecology 70 (5) : 184-195, 2005 (in Japanese)

16. Togari T, Yamazaki Y, Koide S, Miyata A : Reliability and validity of the modified Perceived Health Competence Scale (PHCS) Japanese Version. Japanese Journal of Public Health 53 (1) : 51-57, 2006 (in Japanese)

17. Oshio A, Nakaya M, Kaneko H, Nagamine S : Development and validation of an adolescent resilience scale. Japanese Journal of Counseling Science $35:$ 57-65, 2002 (in Japanese)

18. Fukuhara S, Suzukamo Y : Manual of the SF-8 Japanese version. Institute for Health Outcomes \& Process Evaluation study, Kyoto, 2004 (in Japanese)

19. Fukuhara S, Ware JE, Kosinski M, Wada S, Gandek B : Psychometric and clinical tests of validity of the Japanese SF-36 Health Survey. J Clin Epidemiol 51 : 1045-1053, 1998

20. Izumi H, Saeki K, Imuta H, Mori M : Development of a self-efficacy scale for health behavior for prevention of hyperlipidemia in adults in their 30 s and 40s. The Journal of the Japan Academy of Community Health Nursing 9 (2) : 7-14, 2007 (in Japanese)

21. Ozaki I, Konishi M, Katakura K : Development of self-efficacy scales for middle-aged male workers' health practices -well-balanced diet, physical activity, sleep, drinking behavior, and smoking cessation-. The Journal of the Japan Academy of Community Health Nursing 12 (1) : 87-94, 2009 (in Japanese)

22. Shimizu Y : Relationship between preventive health behavior and self-control of university students. Educational Studies International Christian University 55 : 91-97, 2012 (in Japanese)

23. Elaine NA : Resiliency enhancement-putting the strengths perspective into social work practice. Columbia University Press New York, 2000

24. Adachi M : A study of social work practice based on strengths perspective: As an analysis for the recovery process focusing on "Resiliency" of the person with halfway handicapped. Bulletin of the Graduate School of Social Service Administration Oita University (13) : 1-12, 2010 (in Japanese)

25. Lundman B, Viglund K, Aléx L, Jonsén E, Norberg A, Fischer RS, Strandberg G, Nygren $\mathrm{B}$ : Development and psychometric properties of the Inner Strength Scale. International Journal of Nursing Studies 48 (10) : 1266-1274, 2011

26. Kristi L, Gayle R: Psychometric testing of the Inner Strength Questionnaire : women living with chronic health conditions. Applied Nursing Research 24 : 153-160, 2011

27. Dingley CE, Roux G : Inner strength in older Hispanic women with chronic illness, Journal of Cultural Diversity 10 (1) : 11-22, 2003

28. Lundman B, Aléx L, Jonsén E, Lövheim H, Nygren B, Santamäki Fischer R, Strandberg G, Norberg A : Inner strength in relation to functional status, disease, living arrangements, and social relationships among people aged 85 years and older. Geriatric Nursing 33 (3) : 167176, 2012 